\title{
RESINAS TROCADORAS DE CÁTIONS NA HIPERCALEMIA AGUDA GRAVE
}

\section{CÁTION EXCHANGE RESINS IN SEVERE ACUTE HYPERKALEMIA}

DOI: $10.5380 /$ rmu.v1i3.40751

Maria Aparecida Pachaly ${ }^{1}$

\section{INTRODUÇÃO}

As resinas trocadoras de cátions fazem parte da abordagem terapêutica tradicional da hipercalemia. As resinas de troca catiônica são substâncias sintéticas, não absorvíveis, que liberam um cátion que faz parte de sua composição e captam outro cátion de interesse, no caso, o potássio, causando a redução da calemia. As resinas disponíveis atualmente são: poliestireno sulfonato de sódio (PSS), nos Estados Unidos, e poliestireno sulfonato de cálcio (PSC), no Brasil. Grande parte da literatura encontrada sobre estas resinas é proveniente de países onde é utilizado o PSS, cuja sua estrutura química e efeitos são similares aos do PSC. Porém, como as resinas atualmente disponíveis são efetivas a partir de 24 horas de uso, não atendem à demanda pela remoção rápida de potássio, como ocorre na hipercalemia aguda com toxicidade cardíaca e manifestações eletrocardiográficas. Além disto, apesar de não serem absorvidas, as resinas não são substâncias inócuas, e podem causar complicações graves no aparelho digestivo.

Decisões sobre o uso das resinas na hipercalemia aguda grave devem ser feitas com cautela e considerando o contexto de cada paciente. O objetivo desta revisão é trazer mais dados que permitam ao leitor uma visão crítica sobre o papel real destas substâncias no tratamento da hipercalemia aguda.

\section{HISTÓRICO}

O poliestireno sulfonato é um polímero reticular de ligações cruzadas, e que no caso das resinas de troca catiônica de interesse clínico, possui um grupo sulfonato de carga negativa, que é saturado com sódio (PSS) ou cálcio (PSC) ${ }^{1}$.

Este grupo de medicamentos se desenvolveu a partir da comparação com a argila ("terra sigillata", ou "terra selada"), utilizada como antídoto para vários venenos na Idade Média ${ }^{2}$. A argila é rica em silicatos e óxidos de cálcio e alumínio, e as trocas iônicas são base de seu efeito benéfico².

A partir de 1935 foram desenvolvidas resinas com a capacidade de remover cátions ou ânions de soluções variadas, para uso industrial, e posteriormente para modificações no solo e abrandamento da água. O uso clínico se iniciou em 1945, quando foram usadas resinas trocadoras de íons no tratamento da úlcera péptica ${ }^{2}$. Em 1949, uma resina trocadora de cátions foi utilizada para remover sódio de pacientes com insuficiência cardíaca, e durante este estudo, observouse aumento da excreção fecal de potássio e redução da calemia $^{2}$. Este efeito desencadeou a utilização de resinas com o objetivo principal de remover potássio em situações de insuficiência renal, em uma época em que não havia facilidade para se iniciar uma diálise, cujo desenvolvimento técnico se iniciava.

No início da década de 1950, surgiram os primeiros relatos de sucesso no tratamento de hipercalemia com resinas trocadoras de cátions em insuficiência renal anúrica ${ }^{3,4}$. Em 1959 Palmer e cols. relataram o uso de uma resina no tratamento de 17 casos de hipercalemia, com redução diária de 0,3 mEq/l no potássio sérico ${ }^{5}$. Scherr e cols. ${ }^{6}$ publicaram em 1961 a maior série de pacientes tratados com resina de troca catiônica, em que 32 indivíduos hipercalêmicos com insuficiência renal aguda ou crônica foram tratados com PSS por via oral ou retal, em quantidade entre 10-

1 - Universidade Federal do Paraná - UFPR

Professora Assistente do Departamento de Clínica Médica - UFPR

Contato do Autor / Mail to:

Maria Aparecida Pachaly - map_aly@hotmail.com

Rua General Carneiro, 181 - Alto da Glória, Curitiba - PR, 80060-900 
60g/dia (de acordo com a situação clínica e gravidade da hipercalemia) $)^{6}$ Houve redução dos níveis séricos de potássio entre 0,8-1,0 $\mathrm{mEq}$ (via retal e oral, respectivamente) após $24 \mathrm{~h}$ de tratamento. Uma limitação deste estudo foi o fato de que além da resina, os pacientes receberam dieta pobre em potássio e glicose hipertônica, e alguns receberam também bicarbonato e insulina, medidas que causam redução da hipercalemia ${ }^{6}$.

Com base neste grupo de evidências e em outros estudos com menor número de pacientes, o FDA (Food and Drug Administration) considerou o produto efetivo, aprovando a utilização do Kayexalate ${ }^{\circledR}$ para o tratamento da hipercalemia no final da década de 1950 7. Isto ocorreu antes de o FDA exigir que os fabricantes comprovassem a eficiência do produto para que este tivesse sua comercialização. Devido ao pequeno número de pacientes, metodologia utilizada, e pela falta de comprovação de eficácia em curto espaço de tempo, alguns autores julgam que se hoje em dia o Kayexalate ${ }^{\circledR}$ fosse considerado como nova opção terapêutica para a hipercalemia, não seria aprovado ${ }^{7}$.

\section{MODO DE AÇÃO}

Ao longo de todo o trato gastrintestinal, os cátions nos fluidos intestinais competem pelas valências livres na resina, e íons de maior peso molecular e maior valência possuem preferência na ocupação destes sítios $^{8}$. A concentração do potássio é variável no fluido secretado conforme o nível do tubo digestivo. Nos segmentos proximais, onde o teor de potássio é maior devido à ingestão na dieta, há favorecimento da ligação deste cátion com a resina ${ }^{2}$. No intestino delgado a resina capta sódio ${ }^{8}$ e perde potássio. Ao atingir o cólon proximal, o sódio é deslocado da resina, que então capta potássio em maior quantidade ${ }^{8}$. Este processo continua enquanto a resina progride do cólon para o reto.

Quando uma resina de troca catiônica é adicionada a uma solução eletrolítica (secreção gastrintestinal), ocorre uma distribuição equilibrada de íons entre a resina e o fluido luminal. A presença da resina no intestino introduz um compartimento eletrolítico adicional ao organismo, e é necessário tempo para que ocorra equilíbrio iônico entre os compartimentos $^{8}$. Mesmo quando a concentração de cátions na resina atingiu o máximo para aquele segmento do intestino, ainda existe fluxo de íons entre a resina, o fluido circulante e o pool de cátions do organismo. A concentração final de cátions na resina no intestino depende do tipo da resina, $\mathrm{pH}$ e concentração e propriedades físicas dos diferentes íons no fluido circundante ${ }^{8}$.

Devido ao fato de que o uso de laxativos também causa perda de potássio pelas fezes, é necessário avaliar se o resultado benéfico da resina se deve às suas propriedades intrínsecas, ou se é a associação com laxativos que produz a diminuição do potássio. Emmett e cols. ${ }^{9}$ demonstraram que em indivíduos normais, a adição de sorbitol à resina não aumentou significativamente a quantidade de potássio fecal comparada a outros laxativos. Gruy-Kapral e cols. ${ }^{10}$ compararam a excreção de potássio com a administração isolada de resina ou catártico, e resina combinada ao catártico, e concluíram que as resinas não têm efeito adicional ao uso isolado dos catárticos.

\section{DOSE, CAPACIDADE DE LIGAÇÃO E VIAS DE ADMINISTRAÇÃO}

É difícil definir a quantidade correta de resina a usar em cada caso. Diariamente os processos catabólicos habituais liberam no indivíduo normal cerca de $40 \mathrm{mEq}$ de potássio ao dia, que necessitam ser eliminados; quando a eliminação é prejudicada, ocorre elevação da calemia.

As resinas de troca catiônica possuem capacidade teórica de troca de 3,1 mEq de potássio por grama de resina; porém, metade a dois terços desta capacidade são utilizados para outros cátions (amônio, sódio, magnésio, cálcio, cátions orgânicos, lipídeos e proteínas) ${ }^{4}$. Então, para finalidades práticas, é considerada uma capacidade efetiva de ligação com o potássio de $1 \mathrm{mEq}$ por grama da resina. Seria suficiente o uso de 15-20g, 3-4 vezes ao dia. Porém, em pacientes com catabolismo protéico acentuado por infecção, trauma ou cirurgia, esta quantidade deveria ser recalculada e individualizada ${ }^{11}$. As doses mencionadas acima podem ser insuficientes na remoção de potássio em situações de liberação contínua de potássio no espaço extracelular, por dano tecidual ou infecção ${ }^{6}$.

As resinas podem ser utilizadas por via oral ou via retal. Em seres humanos ocorre secreção ativa de potássio na porção retossigmóide do cólon $^{12}$, em quantidade maior que em outras porções do trato gastrintestinal ${ }^{5}$. Devido a esta característica secretória do cólon terminal, poderia ser esperada uma remoção maior de potássio pela via retal em relação à via oral, mas isto não é observado clinicamente. A via retal possui o inconveniente da dificuldade de administração e o desconforto para o paciente.

\section{EFETIVIDADE / EFICÁCIA}

São poucas e fracas as evidências que sugerem que as resinas são eficazes na redução do potássio sérico. Além disto, o uso das resinas não parece ter eficácia superior ao uso isolado de laxativos ${ }^{13}$.

Sua ação efetiva geralmente ocorre após múltiplas doses e ao longo de vários dias, com efeito retardado, e não há evidências consistentes de 
benefício nas primeiras 12 horas $^{7}$ ou com dose única ${ }^{13}$. A queda dos níveis de potássio com a resina é gradual, e esta queda é muito lenta para a terapia imediata de um paciente que apresenta toxicidade com manifestações $\mathrm{ECG}^{11}$.

Este é um dos grandes questionamentos a respeito do uso das resinas em hipercalemia com toxicidade ao ECG. Nesta situação aguda, além das outras medidas para combater os efeitos da hipercalemia na fibra cardíaca e promover o desvio do potássio para o compartimento intracelular, é necessária a rápida remoção do potássio. As resinas não atendem a esta demanda, e a hemodiálise ainda é o melhor tratamento quando há evidência clínica ou eletrocardiográfica de toxicidade grave pelo potássio, principalmente em pacientes com doença renal aguda ou crônica. As resinas poderiam ser usadas enquanto a hemodiálise é preparada ${ }^{14}$.

Em uma revisão sistemática Cochrane sobre o tratamento da hipercalemia aguda, não foram encontradas evidências consistentes, provenientes de ensaios clínicos randomizados de que as resinas sejam efetivas. Ainda é necessário um estudo bem estruturado para observar sua eficácia ${ }^{15}$.

\section{SEGURANÇA}

Quando o PSS se tornou disponível para uso clínico, era diluído em água. Entretanto, a descrição de opacificações do aparelho digestivo causadas por concreções de cristais da resina gerou a preocupação de que pudessem causar obstruções intestinais. Por este motivo se tornou cada vez mais comum a administração do PSS em uma suspensão com sorbitol hipertônico a 70\%, para promover diarréia osmótica e evitar concreções e impactação colônica ${ }^{7,13}$. Porém, são descritos casos de necrose de cólon associada ao uso da combinação entre PSS e sorbitol ${ }^{7,16-18}$, nos quais cristais de PSS podem ser detectados em peças cirúrgicas, aderidos à mucosa lesada ${ }^{13,18}$. Também pode haver associação com lesões esofágicas e gástricas quando administrado por via oral, podendo provocar sangramento e necrose esofágica ${ }^{19}$.

Em uma revisão sistemática publicada em 2013, Harel e cols. ${ }^{20}$ analisaram 30 relatos de 58 casos de efeitos adversos gastrintestinais, sendo 41 deles envolvendo o uso de PSS com sorbitol e 17 sem sorbitol. O cólon foi o principal local de lesão (76\%) e necrose transmural foi o achado histopatológico mais comum (62\%) nas lesões. A mortalidade nos casos de lesões gastrintestinais foi de $33 \%{ }^{20}$. A maior parte dos casos de necrose do cólon associados à resina ocorre de horas a vários dias após a última dose, e com doses que variaram de 20 a $120 \mathrm{~g}$. Os sintomas geralmente são: dor abdominal, náuseas ou diarréia sanguinolenta ${ }^{21}$. A observação de cristais eosinofílicos de PSS ou PSC em áreas de ulceração sugere 0 diagnóstico ${ }^{21,22}$. A combinação de dados clínicos e histológicos é necessária para excluir causas comuns de necrose da mucosa, e para que se possa atribuir lesões ao uso da resina ${ }^{22}$.

É difícil definir a participação do sorbitol no desenvolvimento da necrose intestinal em pacientes que apresentam múltiplos problemas clínicos e são predispostos a desordens isquêmicas. Um efeito tóxico direto da combinação resina e sorbitol é sugerido pela evidência de necrose de cólon em modelo experimental ${ }^{19,23}$. Acredita-se que o sorbitol seja responsável por alterações hemodinâmicas na parede intestinal, mas o mecanismo não é claro ${ }^{21}$. A condição clínica geral do paciente é importante, pois muitos pacientes que apresentam esta complicação estão hipotensos ou hipovolêmicos, após cirurgia ou diálise ou apresentam doença vascular ${ }^{21}$. A vasculatura intestinal de pacientes renais pode ser predisposta à vasoconstrição pelos níveis elevados de renina $e$ angiotensina ${ }^{24}$. Além disto, a produção de prostaglandinas pode ser estimulada pelo sorbitol e poderia ser uma causa da lesão mucosa ${ }^{25}$. Necrose intestinal também pode ser observada quando a resina não é associada ao sorbitol. Não existem muitos relatos de lesões do trato digestivo com a resina com cálcio ${ }^{22,26-}$ 28.

Apesar do alerta do FDA de que a combinação de sorbitol e PSS pode se associar a necrose de intestino, a combinação continua a ser amplamente prescrita tanto para hipercalemia aguda como crônica nos Estádios Unidos e em outros países ${ }^{29,30}$, e relato de casos de lesões gastrintestinais continuam a aparecer na literatura recente $\mathrm{e}^{30-32}$. Estes autores argumentam que, em pacientes com risco de vida por hipercalemia, não há nenhum papel para o uso de resinas ou diarréia induzida, pois estas medidas levam várias horas para atingir um efeito. Em vez de sorbitol, eles sugerem que são necessários estudos com outros catárticos, que induzam diarréia secretora, como bisacodil ${ }^{30}$. Vale lembrar que o manitol, que em nosso país eventualmente é usado para evitar obstipação pela resina, é um isômero do sorbitol, e efeitos sobre a mucosa intestinal poderiam da mesma forma ocorrer.

Outras complicações do uso das resinas incluem: hipocalcemia (com o PSS), sobrecarga de volume $^{33}$ e hipocalemia ${ }^{34}$. Também não é recomendado o uso da resina por SNG, pelo risco de vômitos e aspiração, com grave pneumonite química ${ }^{11,13,35}$.

\section{EFEITOS COLATERAIS}

Além da baixa palatabilidade, há efeitos colaterais que são comumente descritos com o uso das resinas: náuseas, vômitos, desconforto abdominal. Com o uso disseminado da resina de sódio (PSS), observou-se 
que outro feito colateral comum era a obstipação, e por este motivo o sorbitol (um laxativo osmótico) passou a ser adicionado ao esquema terapêutico. Para facilitar a administração, foi produzida uma formulação de PSS para uso retal, que já continha sorbitol associado. Então, passaram a ser relatados vários casos de necrose e outros tipos de lesão do cólon em pacientes que utilizaram a resina associada ao sorbitol. Com estas evidências, em 2009 o FDA emitiu um alerta a respeito da possibilidade de complicações no trato gastrintestinal, e uma lista de recomendações para evitar as mesmas ${ }^{36}$.

\section{PACIENTES DE ALTO RISCO}

O risco de necrose intestinal é maior quando o PSS em sorbitol for administrado na primeira semana após cirurgia, devido ao íleo pós-operatório e administração de opióides, que também aumentam o risco de íleo. Em uma série de 117 pacientes, dois (1,8\%) desenvolveram necrose intestinal ${ }^{13,16}$. O PSS também não deve ser administrado em pacientes com obstrução do cólon.

\section{RESINA COM CÁLCIO (PSC)}

No Brasil é disponível a resina com cálcio (PSC), que possui a mesma atividade farmacológica que a resina que contém sódio ${ }^{22}$. Ela apresenta a vantagem de liberar cálcio (íon pouco absorvido pelo intestino na falência renal), em troca pelo potássio ${ }^{37}$. Diferente da resina com sódio, não causa expansão do espaço extracelular ou hipernatremia, e pode ser utilizada até em pacientes que estejam em restrição de sódio. Pode ser diluída em água ou glicose a $5 \%{ }^{22}$. No Brasil não existe preparação desta resina contendo sorbitol ou outro laxativo osmótico. Há autores que não recomendam que seja administrada com laxativos osmóticos, pois isto diminuiria a quantidade removida de potássio ${ }^{37}$.

Em um estudo brasileiro com 20 pacientes com doença renal crônica em estágio terminal (DRCET), a resina de cálcio mostrou-se tão eficaz quanto a resina de sódio, mas com a vantagem de não ofertar sódio para troca pelo potássio. Além disto, não foi observada hipercalcemia, e a tolerabilidade à resina foi considerada boa ${ }^{38}$.

\section{RESINAS NA HIPERCALEMIA CRÔNICA}

Em situações menos urgentes, em que é possível aguardar 24 horas para que seus efeitos ocorram, as resinas podem ser utilizadas e são efetivas ${ }^{5}$. Um exemplo disto é a hipercalemia em pacientes que utilizam bloqueio do sistema renina-angiotensina- aldosterona (SRAA) no tratamento da insuficiência cardíaca ou outras condições crônicas, nos quais a hipercalemia muitas vezes impede que se atinja a quantidade ideal de bloqueio do SRAA.

Em um estudo retrospectivo, Chernin e cols. $^{30,39}$ analisaram a eficácia e segurança da administração diária de $15 \mathrm{~g}$ de poliestirenossulfonato de sódio sem sorbitol em 14 pacientes com CKD e doença cardíaca que desenvolveram hipercalemia durante tratamento com IECA, BRA e/ou espironolactona ${ }^{39}$. O potássio médio caiu de $6,4 \pm 0,3$ $\mathrm{mEq} / \mathrm{l}(6,0-7,1 \mathrm{mEq} / \mathrm{l})$ para $4,5 \pm 0,6 \mathrm{mEq} / \mathrm{l}(3,0-5,8$ $\mathrm{mEq} / \mathrm{l})(\mathrm{P}<0,01)$. Hipocalemia discreta $(3,0$ e 3,1 mEq/l) se desenvolveu em 2 pacientes que utilizaram dose reduzida em dias alternados. A droga foi bem tolerada e sem efeitos adversos graves.

\section{AGENTES EXPERIMENTAIS}

Os benefícios limitados e eventuais riscos das resinas de troca catiônica atualmente disponíveis levaram a uma busca por outros agentes para o tratamento de hipercalemia. Pitt e cols. estudaram a eficácia e segurança da RLY5016, um polímero não absorvível experimental para uso por via oral, sobre os níveis séricos de potássio em pacientes recebendo um ACE ou ARB e espironolactona para a insuficiência cardíaca ${ }^{40}$. Durante um estudo de 4 semanas, o potássio sérico de 55 pacientes randomizados para receber RLY016 foi de 0,46 mEq/I menor do que os 49 pacientes randomizados para placebo. Os resultados foram semelhantes em um subgrupo com DRC concomitante. RLY5016 é um polímero sintético, com propriedades otimizadas de fluxo e viscosidade, e não necessita de um catártico para alcançar mais rapidamente o cólon ${ }^{40}$.

Silicato de zircônio é outro "quelante" experimental de potássio que não é sistemicamente absorvido $^{30}$. De acordo com a empresa que o está desenvolvendo, ele parece ser 10x mais potente que o PSS na ligação com o potássio. Um ensaio clínico de fase 2 em 90 pacientes com DRC e hipercalemia leve a moderada foi concluído e um ensaio clínico de fase 3 em um total de 750 pacientes com hipercalemia (potássio sérico 5,0-6,5 $\mathrm{mEq} / \mathrm{l}$ ) encontra-se em andamento em cerca de 75 locais na América do Norte, Europa e Austrália ${ }^{30}$.

\section{RECOMENDAÇÕES}

No alerta de cautela emitido pelo FDA (2009) com relação à resina disponível nos Estados Unidos (PSS), as seguintes recomendações foram feitas: "Casos de necrose intestinal, que podem ser fatais, e outros efeitos adversos gastrintestinais (sangramento, colite isquêmica, perfuração) têm sido relatados em 
associação com o uso de Kayexalate ${ }^{\circledR}$. Não deve ser utilizada em pacientes que não apresentem cólon funcionante. Isto inclui pacientes em pós-operatório que ainda não tenham apresentado evacuações. Não utilizar em pacientes que estejam em risco para o desenvolvimento de obstipação ou impactação fecal (incluindo aqueles com histórico de impactação, obstipação crônica, doença inflamatória intestinal, colite isquêmica, aterosclerose vascular intestinal, ressecção prévia do cólon, ou obstrução do cólon). Interromper o uso em pacientes que desenvolvem obstipação. Não administrar doses repetidas em pacientes que não estão evacuando" ${ }^{36}$.

Além destas recomendações e pelos mecanismos fisiopatológicos envolvidos na necrose intestinal, considerar com cuidado sua utilização em pacientes com instabilidade hemodinâmica, em uso de aminas vasoativas em doses altas; doenças avançadas de outros órgãos e sistemas; distensão abdominal intensa ou ascite volumosa; síndrome compartimental abdominal; depleção de volume e status pós parada cardiorrespiratória. Estas considerações podem soar paradoxais, pois são justamente as características de muitos dos pacientes que desenvolvem hipercalemia grave. Porém, deve existir reflexão sobre a necessidade do uso das resinas e a melhor estratégia, dada sua baixa efetividade e as questões de segurança. Os médicos devem estar cientes do risco de efeitos adversos, especialmente no subgrupo de pacientes que possuem múltiplos problemas clínicos. A terapia da hipercalemia aguda com resinas de troca é considerada potencialmente prejudicial e sem eficácia comprovada 7 . Para seu uso ser justificado, os argumentos devem ser muito consistentes.

Além disto, como atualmente o acesso à diálise é rápido, a resina não deve ser vista como forma de postergar o procedimento dialítico em pacientes com insuficiência renal.

\section{CONCLUSÕES}

\begin{abstract}
O papel das resinas trocadoras de íons na hipercalemia aguda com manifestação eletrocardiográfica é questionável, pois o tempo necessário para a ação da resina é incompatível com a rapidez necessária nesta situação que ameaça à vida. Além disto, há complicações graves que devem ser consideradas.
\end{abstract}

\section{REFERÊNCIAS BIBLIOGRÁFICAS}

1. Kamel, K.S. and M. Schreiber, Asking the question again: are cation exchange resins effective for the treatment of hyperkalemia? Nephrol Dial Transplant, 2012. 27(12): p. 4294-7.

2. Irwin, L., E.Y. Berger, and et al., The effect of a cation exchange resin on electrolyte balance and its use in edematous states. J Clin Invest, 1949. 28(6 Pt 2): p. 1403-11.
3. Elkinton, J.R., et al., Treatment of potassium retention in anuria with cation exchange resin; a preliminary report. Am J Med Sci, 1950. 220(5): p. 547-52.

4. Evans, B.M., et al., lon-exchange resins in the treatment of anuria. Lancet, 1953. 265(6790): p. 791-5.

5. Palmer, R.A., J.D. Price, and J. Eden, The treatment of hyperkalaemia by carboxylic acid resins in the upper and lower gastrointestinal tract. Can Med Assoc J, 1959. 80(6): p. 432-5.

6. Scherr, L., et al., Management of hyperkalemia with a cationexchange resin. N Engl J Med, 1961. 264: p. 115-9.

7. Sterns, R.H., et al., lon-exchange resins for the treatment of hyperkalemia: are they safe and effective? J Am Soc Nephrol, 2010. 21(5): p. 733-5.

8. Spencer, A.G., E.J. Ross, and H.G. Lloyd-Thomas, Cation exchange in the gastrointestinal tract. Br Med J, 1954. 1(4862): p. 603-6.

9. Emmett, M., et al., Effect of three laxatives and a cation exchange resin on fecal sodium and potassium excretion. Gastroenterology, 1995. 108(3): p. 752-60.

10. Gruy-Kapral, C., et al., Effect of single dose resin-cathartic therapy on serum potassium concentration in patients with endstage renal disease. J Am Soc Nephrol, 1998. 9(10): p. 1924-30.

11. Flinn, R.B., J.P. Merrill, and W.R. Welzant, Treatment of the oliguric patient with a new sodium-exchange resin and sorbitol; a preliminary report. N Engl J Med, 1961. 264: p. 111-5.

12. Sandal, S., et al., To bind or to let loose: effectiveness of sodium polystyrene sulfonate in decreasing serum potassium. Int J Nephrol, 2012. 2012: p. 940320.

13. Mount, D.B., Treatment and prevention of hyperkalemia in adults. , in UpToDate, R.H. Sterns and J.P. Forman, Editors. 2014, Wolters Kluwer: Waltham, MA. p. 21.

14. Watson, M., K.C. Abbott, and C.M. Yuan, Damned if you do, damned if you don't: potassium binding resins in hyperkalemia. Clin J Am Soc Nephrol, 2010. 5(10): p. 1723-6.

15. Mahoney, B.A., et al., Emergency interventions for hyperkalaemia. Cochrane Database Syst Rev, 2005(2): p. CD003235.

16. Gerstman, B.B., R. Kirkman, and R. Platt, Intestinal necrosis associated with postoperative orally administered sodium polystyrene sulfonate in sorbitol. Am J Kidney Dis, 1992. 20(2): p. 159-61.

17. Lillemoe, K.D., et al., Intestinal necrosis due to sodium polystyrene (Kayexalate) in sorbitol enemas: clinical and experimental support for the hypothesis. Surgery, 1987. 101(3): p. 267-72.

18. McGowan, C.E., et al., Intestinal necrosis due to sodium polystyrene sulfonate (Kayexalate) in sorbitol. South Med J, 2009. 102(5): p. 493-7.

19. Abraham, S.C., et al., Upper gastrointestinal tract injury in patients receiving kayexalate (sodium polystyrene sulfonate) in sorbitol: clinical, endoscopic, and histopathologic findings. Am J Surg Pathol, 2001. 25(5): p. 637-44.

20. Harel, Z., et al., Gastrointestinal adverse events with sodium polystyrene sulfonate (Kayexalate) use: a systematic review. Am J Med, 2013. 126(3): p. 264 e9-24.

21. Kelsey, P.B., S. Chen, and G.Y. Lauwers, Case records of the Massachusetts General Hospital. Weekly clinicopathological exercises. Case 37-2003. A 79-year-old man with coronary artery disease, peripheral vascular disease, end-stage renal disease, and abdominal pain and distention. N Engl J Med, 2003. 349(22): p. 2147-55.

22. Joo, M., et al., Colonic mucosal necrosis following administration of calcium polystryrene sulfonate (Kalimate) in a uremic patient. J Korean Med Sci, 2009. 24(6): p. 1207-11.

23. Wootton, F.T., et al., Colonic necrosis with Kayexalate-sorbitol enemas after renal transplantation. Ann Intern Med, 1989. 111(11): p. 947-9.

24. Reilly, P.M. and G.B. Bulkley, Vasoactive mediators and splanchnic perfusion. Crit Care Med, 1993. 21(2 Suppl): p. S5568.

25. Zijlstra, F.J., Sorbitol, prostaglandins, and ulcerative colitis enemas. Lancet, 1981. 2(8250): p. 815-6. 


\section{REVISTA MÉDICA DA UFPR}

26. Lee, S.H., et al., [Calcium polystyrene sulfonate induced colonic necrosis in patient with chronic kidney disease]. Korean J Gastroenterol, 2010. 55(4): p. 261-5.

27. Shioya, T., et al., Successful treatment of a colonic ulcer penetrating the urinary bladder caused by the administration of calcium polystyrene sulfonate and sorbitol. J Nippon Med Sch, 2007. 74(5): p. 359-63.

28. Goutorbe, P., et al., Intestinal Necrosis Associated with Orally Administered Calcium Polystyrene Sulfonate Without Sorbitol (February). Ann Pharmacother, 2011.

29. Fordjour, K.N., T. Walton, and J.J. Doran, Management of hyperkalemia in hospitalized patients. Am J Med Sci, 2014. 347(2): p. 93-100.

30. Sterns, R.H. and M. Emmett, Fluid, electrolyte, and Acid-Base Disturbances. Nephrology Self-Assessment Program (NephSAP) American Society of Nephrology - Journal of the American Society of Nephrology, 2013. 12(3)

31. Chelcun, J.L., R.A. Sable, and K. Friedman, Colonic ulceration in a patient with renal disease and hyperkalemia. JAAPA, 2012. 25(10): p. 34, 37-8.

32. Gorospe, E.C., J.T. Lewis, and D.H. Bruining, Kayexalate-induced esophageal ulcer in a patient with gastroparesis. Clin Gastroenterol Hepatol, 2012. 10(5): p. A28.

33. Berlyne, G.M., K. Janabi, and A.B. Shaw, Dangers of resonium A in the treatment of hyperkalemia in renal failure. Lancet, 1966. 1(7430): p. 167-9.
34. Gales, M.A., et al., Rectally administered sodium polystyrene sulfonate. Am J Health Syst Pharm, 1995. 52(24): p. 2813-5.

35. Idowu, M.O., M. Mudge, and N.R. Ghatak, Kayexalate (sodium polystyrene sulfonate) aspiration. Arch Pathol Lab Med, 2005. 129(1): p. 125.

36. Administration., U.F.a.D. Safety Warning - Kayexalate (sodium polystyrene sulfonate) powder. [Electronic] 2009 January 2011 [cited 2014 June 16, 2014]; Available from: http://www.fda.gov/Safety/MedWatch/SafetyInformation/ucm 186845.htm.

37. Berlyne, G.M., et al., Treatment of hyperkalemia with a calciumresin. Lancet, 1966. 1(7430): p. 169-72.

38. Loureiro, A.A., L.E. lanhez, and E. Sabbaga, [Clinical evaluation of a cation-exchange resin (calcium Serdolit) in the treatment and prevention of hyperkalemia]. Rev Hosp Clin Fac Med Sao Paulo, 1972. 27(6): p. 267-72.

39. Chernin, G., et al., Secondary prevention of hyperkalemia with sodium polystyrene sulfonate in cardiac and kidney patients on renin-angiotensin-aldosterone system inhibition therapy. Clin Cardiol, 2012. 35(1): p. 32-6.

40. Pitt, B., et al., Evaluation of the efficacy and safety of RLY5016, a polymeric potassium binder, in a double-blind, placebocontrolled study in patients with chronic heart failure (the PEARL-HF) trial. Eur Heart J, 2011. 32(7): p. 820-8 